\title{
Dilemmas in resuscitation of COVID-19 patients based on current evidence
}

\author{
Lukasz Szarpak ${ }^{1}$, Kurt Ruetzler ${ }^{2}$, Marek Dabrowski ${ }^{3}$, Klaudiusz Nadolny ${ }^{4}$, \\ Jerzy R. Ladny ${ }^{5}$, Jacek Smereka ${ }^{6}$, Milosz Jaguszewski ${ }^{7}, K_{r} z y s z t o f$ J. Filipiak ${ }^{8}$ \\ ${ }^{1}$ Lazarski University, Warsaw, Poland; Polish Society of Disaster Medicine, Warsaw, Poland \\ ${ }^{2}$ Departments of Outcomes Research and General Anesthesia, Anesthesiology Institute, \\ Cleveland Clinic, Cleveland, Ohio, United States \\ ${ }^{3}$ Chair and Department of Medical Education, Poznan University of Medical Sciences, Poznan, Poland \\ ${ }^{4}$ Department of Emergency Medical Service, Higher School of Strategic \\ Planning in Dabrowa Gornicza, Dabrowa Gornicza, Poland \\ ${ }^{5}$ Clinic of Emergency Medicine, Medical University of Bialystok, Poland; \\ Polish Society of Disaster Medicine, Warsaw, Poland \\ ${ }^{6}$ Department of Emergency Medical Service, Wroclaw Medical University, Wroclaw, Poland; \\ Polish Society of Disaster Medicine, Warsaw, Poland \\ ${ }^{7} 1^{\text {st }}$ Department of Cardiology, Medical University of Gdansk, Poland \\ ${ }^{8} 1^{\text {st }}$ Chair and Department of Cardiology, Medical University of Warsaw, Poland
}

This paper was guest edited by Prof. Łukasz K. Czyżewski

The authors of the present article read a recent article by Shao et al. [1] published in the Resuscitation Journal with interest. Indeed, since the beginning of the severe acute respiratory syndrome coronavirus 2 (SARS-CoV-2) epidemic, there have been $2,435,876$ infected patients confirmed, with an observed level of mortality of nearly $8 \%$. The study by Shao et al. [1] is of considerable importance as it is the first to present the survival of COVID-19 patients after in-hospital cardiac arrest. The authors both characterized the patients and compared a number of factors that may affect the return of spontaneous circulation, as well as 30-day survival.

In an extreme situation, such as a prevailing pandemic, extraordinary methods should be applied [2]. The decisions taken may often conflict with the general principle of "first do no harm" and with its intrinsic ethical values; however, medical personnel should take into account not only their patient, but also their own safety, as well as the safety of their other patients, families, and colleagues [3]. As in the case of SARS and MERS, the primary route of spreading COVID-19 is the droplet or direct contact pathway $[4,5]$. It is worth remembering that HCoV-SARS, HCoV-MERS, and SARS-CoV-2 can also be transmitted via environmental vectors [6]. Therefore, when contacting a patient with suspected or, particularly, confirmed COVID-19, medical personnel should use personal protective equipment for aerosol generating procedures and all procedures regarding such a patient, including resuscitation, which should be performed in airborne infection isolation rooms.

Wang et al. [7] indicated that the time from the disease onset to death was shorter in people over 70 years of age compared with younger patients (11.5 and 20 days, respectively). Yang et al. [8] observed that in COVID-19 patients, comorbidities and diagnosed underlying diseases, including hypertension and respiratory system and cardiovascular diseases, may constitute risk factors for severe compared with the non-severe course of COVID-19. These results were confirmed in a study by Shao et al. [1], in which cardiac arrest

Address for correspondence: Lukasz Szarpak, Assoc. Prof. PhD, Lazarski University, ul. Świeradowska 43, 02-662 Warszawa, Poland, tel: +48 500186225, e-mail: lukasz.szarpak@gmail.com 
in people over 60 years of age was associated with a higher risk of death; similarly, the risk of hypertension, the most common coexisting disease in this group, increased with age. The higher risk of death in the elderly with COVID-19 may be associated with impaired immune response, which is further reduced by the effect of coronavirus.

Shao et al. [1] also referred to two important issues. Firstly, patients with COVID-19 pneumonia should be monitored in intensive care units, which, compared with general wards, provide a higher chance of survival. Secondly, the survival of patients with non-shockable rhythms is below $0.8 \%$; this, considered herein, is the most important concern. In this context, a question arises whether cardiopulmonary resuscitation should be conducted in each patient with COVID-19, exposing medical personnel and their closest associates, or, in the case of elderly patients with initial non-shockable rhythms, resuscitation should not be undertaken. The answer to this question should be established through discussions in individual therapeutic teams.

Conflict of interest: None declared

\section{References}

1. Shao F, Xu S, Ma X, et al. In-hospital cardiac arrest outcomes among patients with COVID-19 pneumonia in Wuhan, China. Resuscitation. 2020 [Epub ahead of print]; 151: 18-23, doi: 10.1016/j. resuscitation.2020.04.005, indexed in Pubmed: 32283117.

2. Dzieciatkowski T, Szarpak L, Filipiak KJ, et al. COVID-19 challenge for modern medicine. Cardiol J. 2020; 27(2): 175-183, doi: 10.5603/CJ.a2020.0055, indexed in Pubmed: 32286679.

3. Ruetzler K, Szarpak L, Filipiak K, et al. The COVID-19 pandemic - a view of the current state of the problem. Disaster Emerg Med J. 2020, doi: 10.5603/demj.a2020.0015.

4. Smereka J, Szarpak L, Filipiak K. Modern medicine in COVID-19 era. Disaster Emerg Med J. 2020, doi: 10.5603/demj.a2020.0012.

5. Szarpak L, Smereka J, Filipiak KJ, et al. Cloth masks versus medical masks for COVID-19 protection. Cardiol J. 2020; 27(2): 218-219, doi: 10.5603/CJ.a2020.0054, indexed in Pubmed: 32285928.

6. Smereka J, Szarpak L. The use of personal protective equipment in the COVID-19 pandemic era. Am J Emerg Med. 2020 [Epub ahead of print], doi: 10.1016/j.ajem.2020.04.028, indexed in Pubmed: 32305157.

7. Wang W, Tang J, Wei F. Updated understanding of the outbreak of 2019 novel coronavirus (2019-nCoV) in Wuhan, China. J Med Virol. 2020; 92(4): 441-447, doi: 10.1002/jmv.25689, indexed in Pubmed: 31994742.

8. Yang J, Zheng Ya, Gou Xi, et al. Prevalence of comorbidities in the novel Wuhan coronavirus (COVID-19) infection: a systematic review and meta-analysis. Int J Infect Dis. 2020 [Epub ahead of print]; 94: 91-95, doi: 10.1016/j.ijid.2020.03.017, indexed in Pubmed: 32173574. 